\title{
COMPARAISON ENTRE LA DIAPAUSE LARVAIRE CHEZ ANABOLIA NERVOSA CURTIS ET HALESUS RADIATUS CURTIS [TRICHOPTERA, LIMNEPHILIDAE]
}

\author{
par C. DENIS 1
}

\begin{abstract}
Par l'étude de la chronologie des comportements et de l'évolution organique à la fin de la vie larvaire des deux espèces ainsi que par l'étude de l'action d'une photopériode de 18 heures sur les larves âgées de $H$. radiatus, il est établi que chez $A$. nervosa, la diapause se situe à la fin de la croissance larvaire, avant la métamorphose et stoppe le développement. Chez $H$. radiatus, elle intervient soit à la fin de la croissance larvaire, soit encore au début de la métamorphose, et le plus souvent elle ne fait que ralentir le développement.
\end{abstract}

\section{A comparison between the larval diapause in Anabolia nervosa Curtis and Halesus nadiatus Curtis (Trichoptera - Limnephilidae).}

By studying the chronology of the behaviour and the organic evolution of both speoies, at the end of their larval life and also the aotion of an $18 \mathrm{~h}$ long photoperiod on old larvae of $H$. radiatus, we have established that with $A$. nervosa, the diapause is situated at the end of the larval growth periode, before metamorphosis and the cessation of the development. With $H$. radiatus, it intervenes either at the end of the larval growth or else at the beginning of metamorphosis and it often merely reduces the rate of the development.

\section{1. - INTRODUCTION}

Les larves de Trichoptères parvenues au terme de leur croissance obturent par un opercule soyeux l'orifice antérieur, puis l'orifice postérieur de leur fourreau quelques jours avant la mue nymphale. Chez Anabolia nervosa et Halesus radiatus qui terminent leur croissance en juin mais subissent une diapause sous l'action de la photopériode longue des jours d'été (Denis, 1972 et 1973), l'obturation du fourreau n'intervient qu'en fin août ou en septembre, juste avant la métamorphose. C'est du moins la règle générale pour $A$. nervosa. Chez $H$. radiatus, si c'est également le cas pour certaines larves, beaucoup d'autres construisent le premier opercule de leur fourreau dès le mois de juin, bien que le second le soit toujours en septembre. Il existe donc des différences individuelles dans le comportement des larves de $H$. radiatus. Ces différences peuvent être dues :

1. Laboratoire de Biologie Animale $1^{\text {er }}$ Cycle, U.E.R. Sciences Biologiques, avenue du Général-Leclerc, 35031 Rennes Cedex. 
- soit à une variabilité de la place de la diapause,

- soit à une éventuelle évolution des animaux pendant la diapause,

- soit à une variabilité dans l'état d'évolution des larves lors de la construction du premier opercule du fourreau.

Or, contrairement aux larves de $A$. nervosa qui entrent en diapause aussi bien sous une photopériode hivernale qu'estivale, celles de $H$. radiatus se métamorphosent directement lorsqu'elles terminent leur croissance au début du printemps, avant que la durée de la photopériode ne devienne excessive. Ceci a été obtenu au laboratoire (Denis, 1973). Il se pourrait alors que selon la vitesse de leur développement dans la nature, les larves de cette espèce atteignent un état d'évolution plus ou moins avancé avant d'entrer en diapause.

Dans ce travail, nous nous sommes proposé de déterminer si les larves de $A$. nervosa et de $H$. radiatus étaient en diapause pendant la même étape ou non de leur cycle et de rechercher les causes des différences individuelles constatées chez la seconde espèce. Dans ce but, nous avons entrepris une étude chronologique des étapes du comportement et suivi l'évolution organique des animaux à la fin de leur vie larvaire. En outre, nous avons examiné chez $H$. radiatus les conséquences du passage d'une photopériode courte à une photopériode longue, à partir d'étapes différentes de la fin de leur vie larvaire.

\section{2. - MATERIELS ET METHODES}

Anabolia nervosa Curtis provient de l'Illet, rivière affluente de l'Ille et Halesus radiatus Curtis, d'un ruisseau de la forêt de Rennes. Des animaux récoltés à l'état larvaire se sont métamorphosés et on pondu au laboratoire. Les élevages nécessaires à ce travail ont été réalisés à partir des pontes ainsi obtenues.

Les dispositifs d'élevage et d'expérimentation ont déjà été décrits (Allegret et Denis, 1972 et Denis, 1972). Dans toutes les expériences, les larves ont été maintenues à la température de $15^{\circ} \mathrm{C}$.

L'état d'évolution des animaux a été contrôlé par l'examen, sous la loupe binoculaire, des ébauches de l'appareil génital.

\section{3. - ETUDE DU COMPORTEMENT DES ANIMAUX A LA FIN DE LA VIE LARVAIRE}

\subsection{Les différentes étapes du comportement}

$\mathrm{Au}$ cours du $5^{\mathrm{e}}$ et dernier stade larvaire, lorsque la période d'alimentation est terminée, l'animal cesse d'agrandir son fourreau. Ce der- 


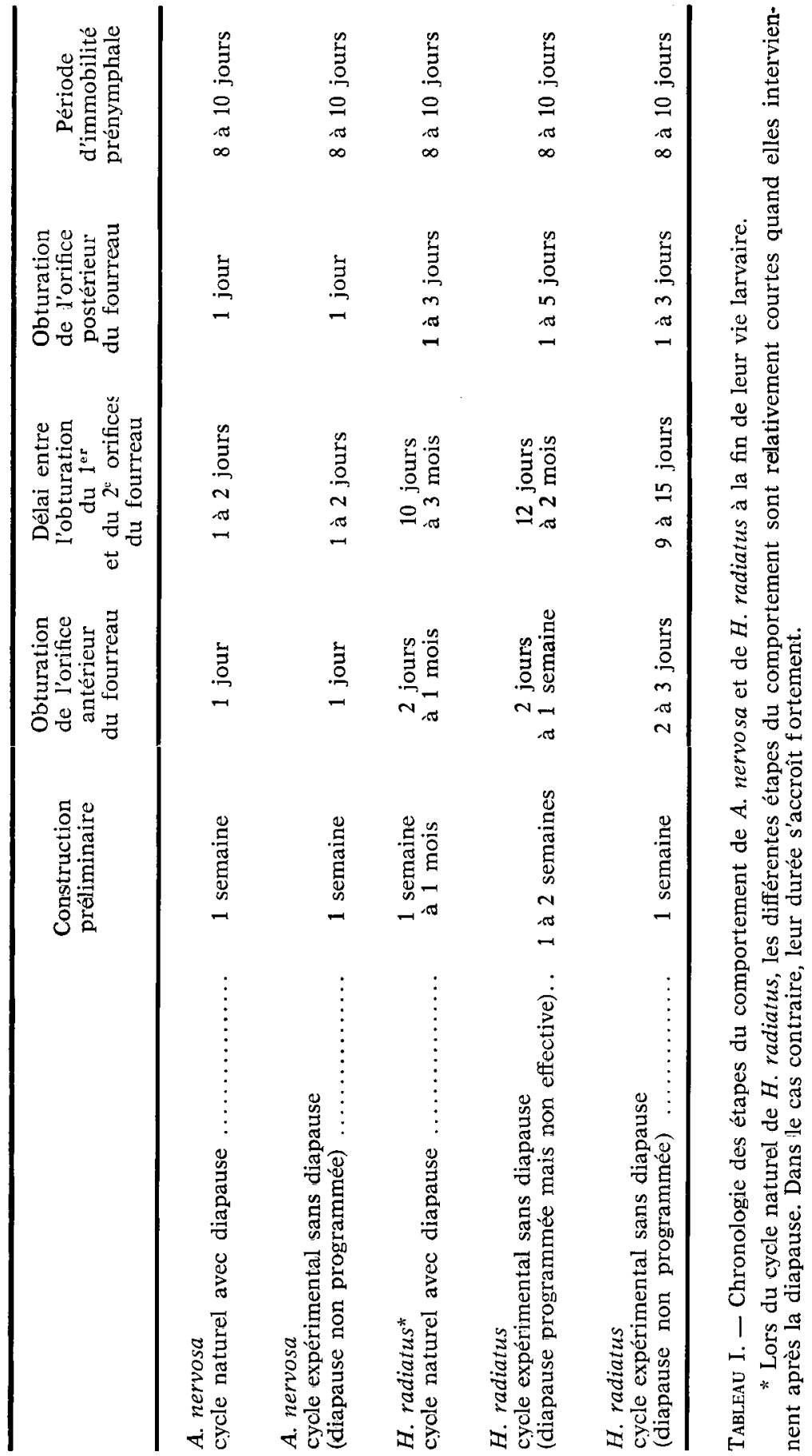


nier présente alors une ouverture antérieure biseautée dont le bord est régularisé par un dépôt soyeux (fig. 1 a et $1 \mathrm{f}$ ). L'orifice postérieur, transversal, est à demi obturé par un disque de soie. Ultérieurement, une reprise de la construction annonce la confection de la logette nymphale dont la réalisation donne lieu à différentes phases de comportement que nous regrouperons en cinq étapes principales comprenant chacune plusieurs actions successives :

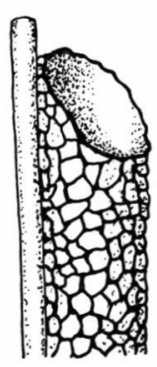

A

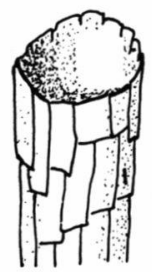

$F$

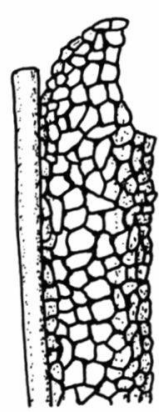

B

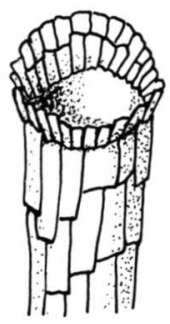

G

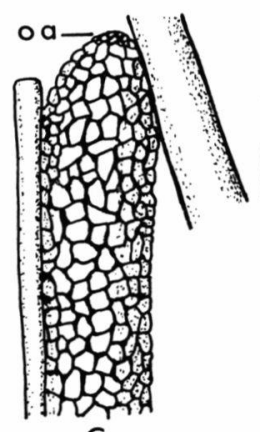

C

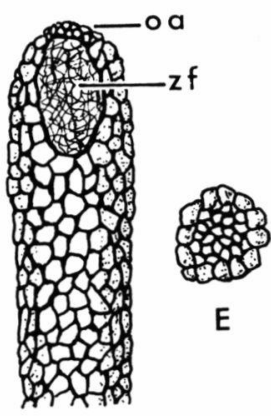

D

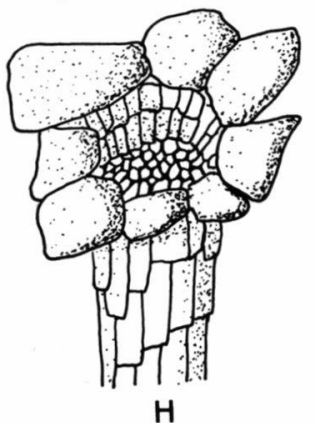

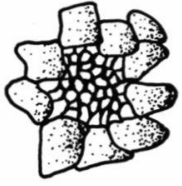

I

Fig. 1. - Les différentes étapes de l'évolution du fourreau pendant la réalisation de la logette nymphale. a, b, c, d, e : fourreau de $A$. nervosa; f, g, h, i : fourreau de $H$. radiatus. a (vue de profil) et $\mathbf{f}$ (vue ventrale) : fourreau en fin de croissance ; b (vue de profil) et $g$ (vue ventrale) : construction préliminaire; $c$ (vue de profil) : fourreau fixé à un support et obturé; d (vue ventrale) : zone de fixation; et et $\mathrm{i}$ : grilles postérieures.

oa: grille antérieure du fourreau; zf : zone de fixation.

I. Construction préliminaire. C'est une nouvelle et dernière phase d'allongement du fourreau.

II. Obturation de l'orifice antérieur du fourreau.

- Ancrage du fourreau par son extrémité antérieure,

- Confection d'une grille de soie au niveau de l'orifice antérieur.

III. Période d'orientation inverse de la larve dans son fourreau. C'est le délai entre l'obturation du premier et du second orifice du fourreau. 
- Tête à queue de l'animal,

- Elimination de l'extrémité postérieure du fourreau.

L'animal restera orienté vers l'arrière de son étui jusqu'à la fin de l'étape suivante.

IV. Obturation de l'orifice postérieur du fourreau.

- Remodelage et, éventuellement, ancrage de l'extrémité postérieure du fourreau,

- Confection d'une grille de soie au niveau de l'orifice postérieur.

V. Période d'immobilité prénymphale. Nouveau tête-à-queue de l'animal qui reprend définitivement son orientation habituelle dans le fourreau.

Peu après, la tête et les pattes s'immobilisent; seuls persistent les mouvements ondulatoires de l'abdomen.

La réalisation de la logette nymphale revêt des modalités interspécifiquement variables :

$A$. nervosa construit, au cours de la première étape, un vaste surplomb dorsal dont l'extrémité s'infléchit (fig. 1 b) ; au début de la seconde étape, il fixe son fourreau par la région ventrale (fig. $1 \mathrm{c}$ et $1 \mathrm{~d})$. L'obturation de l'extrémité postérieure du fourreau est rarement précédée d'un ancrage (fig. $1 \mathrm{e}$ ).

$H$. radiatus construit, au cours de la première étape, un bord évasé (fig. $1 \mathrm{~g}$ ) ; au début de la seconde, il ancre l'avant de son fourreau et fixe des éléments minéraux ou végétaux tout autour de l'extrémité antérieure. La grille d'obturation est tissée à la base de la partie évasée (fig. $1 \mathrm{~h}$ ). Enfin, il ancre l'extrémité postérieure de son fourreau et l'entoure également de fragments variés avant de confectionner la seconde grille (fig. $1 \mathrm{i}$ ).

\subsection{Chronologie des étapes du comportement}

Dans notre étude chronologique, nous avons examiné pour chaque espèce non seulement le cas du cycle normal avec diapause, mais également, à titre comparatif, celui où la diapause n'est pas programmée (larves élevées sous photopériode longue) et, chez $H$. radiatus, celui où la diapause, bien que programmée (larves élevées sous photopériode courte, comme dans la nature), n'est pas effective (Denis, 1972 et 1973). Les résultats sont consignés dans le tableau I.

L'étude des étapes successives du comportement des animaux à la fin de leur vie larvaire fait apparaître des différences importantes entre les deux espèces :

- Chez A. nervosa, la chronologie de ces étapes est définie dès le 
début de la construction de la grille antérieure du fourreau. Chez $H$. radiatus, cette chronologie demeure variable jusqu'à la construction de la grille postérieure du fourreau; la seule exception concerne les larves élevées sous photopériode longue, dont la diapause n'a pas été programmée et pour lesquelles la chronologie du développement est définie dès la construction de la grille antérieure du fourreau, comme chez A. nervosa.

- Tandis que les larves de A. nervosa confectionnent leur logette nymphale en trois ou quatre jours seulement, celle de $H$. radiatus mettent au minimum deux semaines et souvent jusqu'à trois mois pour effectuer le même travail.

- Après la réalisation complète de la logette nymphale, la chronologie du développement devient identique chez les deux espèces.

Les différences essentielles entre les cycles des deux espèces sont illustrées par la figure 2 .

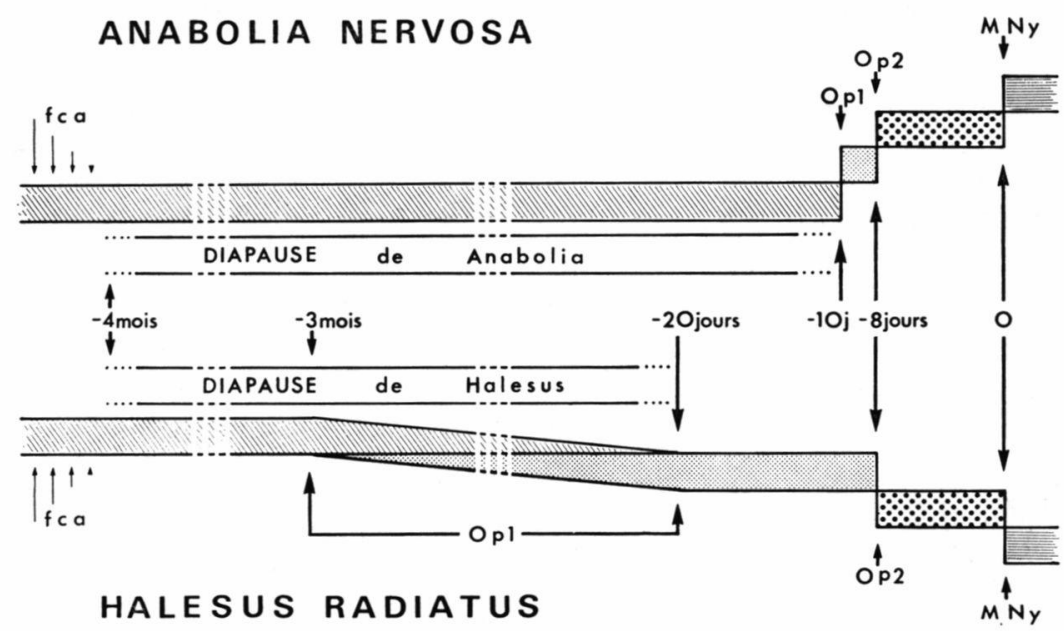

FIG. 2. - Graphique illustrant la chronologie des étapes du cycle naturel des deux espèces à la fin de la vie larvaire. fca : fin de la croissance et de l'alimentation larvaires; op1 et op2: moment de la construction du $1^{\text {er }}$ et du $2^{\text {e }}$ opercules du fourreau; MNy : mue nymphale.

\section{4. - L'EVOLUTION ORGANIQUE}

Elle a été suivie par l'examen des ébauches de l'appareil génital lors des étapes caractéristiques de la fin de la vie larvaire et à la mue nymphale. Cet examen a porté sur des animaux dont les conditions de développement correspondaient aux différents cas indiqués dans le tableau I. 


\section{RÉSULTATS (fig. 3)}

Chez les deux espèces et quelles que soient les conditions du développement des animaux, nous avons constaté que les ébauches de l'appareil génital apparaissaient à la fin de l'étape dite : " construction préliminaire ». Dans le cas du mâle que nous avons choisi d'exposer, l'évolution est la suivante :

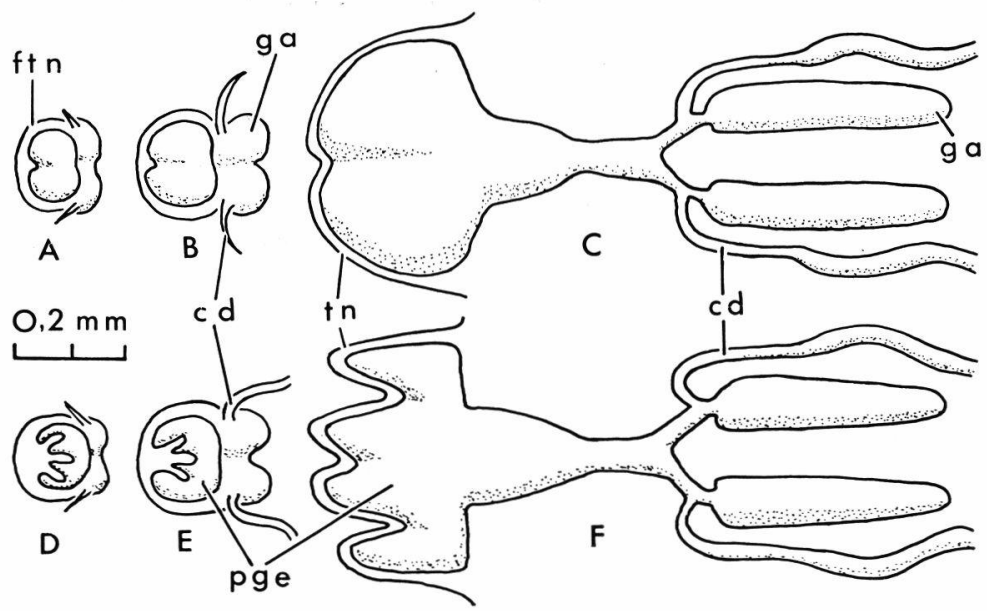

Fig. 3. - Evolution des ébauches de l'appareil génital mâle. a, b et c: $H$. radiatus; d, e, f : A nervosa. a et d: lors de la construction de la grille antérieure; $\mathrm{b}$ et $\mathrm{e}$ : lors de la construction de la grille postérieure; $\mathrm{c}$ et $\mathrm{f}$ : à la mue nymphale.

cd: canal déférent; ga: glande accessoire; ftn : futur tégument nymphal ; pge : pièces génitales externes; tn : tégument nymphal.

- Lors de la confection de la grille antérieure du fourreau les ébauches des pièces génitales externes sont nettement visibles et, en avant de celles-ci, deux légers reliefs apparaissent, qui marquent l'origine des glandes accessoires. Deux condons courts s'en écartent, ills sont dirigés tout d'abond vers l'arrière du corps; ills correspondent aux futurs canaux déférents ( $f i g .3$ a et 3 d).

Notons que chez $H$. radiatus, ces ébauches sont toujours présentes, et au même état d'évolution, lors de la confection de la grille antérieure du fourreau, que celle-ci ait lieu après la diapause (en septembre) ou avant (en juin).

- Lors de la confection de la grille postérieure du fourreau, les ébauches des glandes accessoires forment deux lobes nettement renflés et celles des canaux déférents se sont allongées et incurvéee vers l'avant du corps (fig. 3 b et 3 e).

- A la mue nymphale, les ébauches ont beaucoup grandi : celles des glandes accessoires sont tubulaires et celles des canaux déférents se sont développées en direction des testicules (fig. $3 \mathrm{c}$ et $3 \mathrm{f}$ ). 
Les différentes étapes du comportement à la fin de la vie larvaire correspondent donc à des états d'évolution organique définis et identiques dans tous les cas chez les deux espèces.

\section{5. - ACTION D'UNE PHOTOPERIODE LONGUE SUR DES LARVES AGEES DE H. RADIATUS}

Des larves ayant effectué tout leur développement sous photopériode courte $(12 \mathrm{~h})$ ont été soumises à l'action d'une photopériode longue $(18 \mathrm{~h})$ avant ou après le début de l'obturation du fourreau. Trois lots ont été iconstitués :

- Lot 1: 30 larves à la fin de leur croissance (donc avant l'apparition des ébauches des organes imaginaux).

- Lot 2: 22 larves en train de construire l'évasement du bord antérieur du fourreau (donc très peu avant ou très peu après l'apparition des ébauches des organes imaginaux).

- Lot 3: 26 larves ayant construit depuis un à trois jours la grille antérieure du fourreau (les ébauches des organes imaginaux ont donc commencé à se développer).

Les lots constitués fin mars ont été maintenus sous la même photopériode : $18 \mathrm{~h}$ jusqu'à l'arrêt des expériences le 31 juillet. Les résultats sont consignés dans le tableau II.

Par rapport aux données fournies par des larves laissées sous photopériode courte (tableau I, 4e ligne), les résultats obtenus avec les lots 1 et 2 font apparaître une retard important dans la réalisation de la grille antérieure du fourreau, retard dû manifestement à l'augmentation de la photopériode. De même, tandis que dans la nature, la grille postérieure du fourreau est construite en un à trois jours (tableau $\mathrm{I}, 3^{\mathrm{e}}$ ligne), sa réalisation peut demander un mois dans nos expériences (tableau II). La différence tient sans aucun doute au fait que, dans la nature, l'évolution des larves est accélérée en automne par la diminution de la photopériode qui rompt leur diapause, tandis que dans nos expériences, les animaux sont maintenus dans les conditions de la diapause.

Ces expériences montrent que, chez $H$. radiatus, l'action de la photopériode longue :

- peut s'exercer à des moments différents du cycle : avant ou après la confection de la grille antérieure du fourreau - c'est-à-dire avant ou après l'apparition des ébauches des organes imaginaux - et jusqu'à la réalisation complète de la logette nymphale,

- stoppe ou du moins ralentit considérablement les processus de la métamorphose, 
(9)

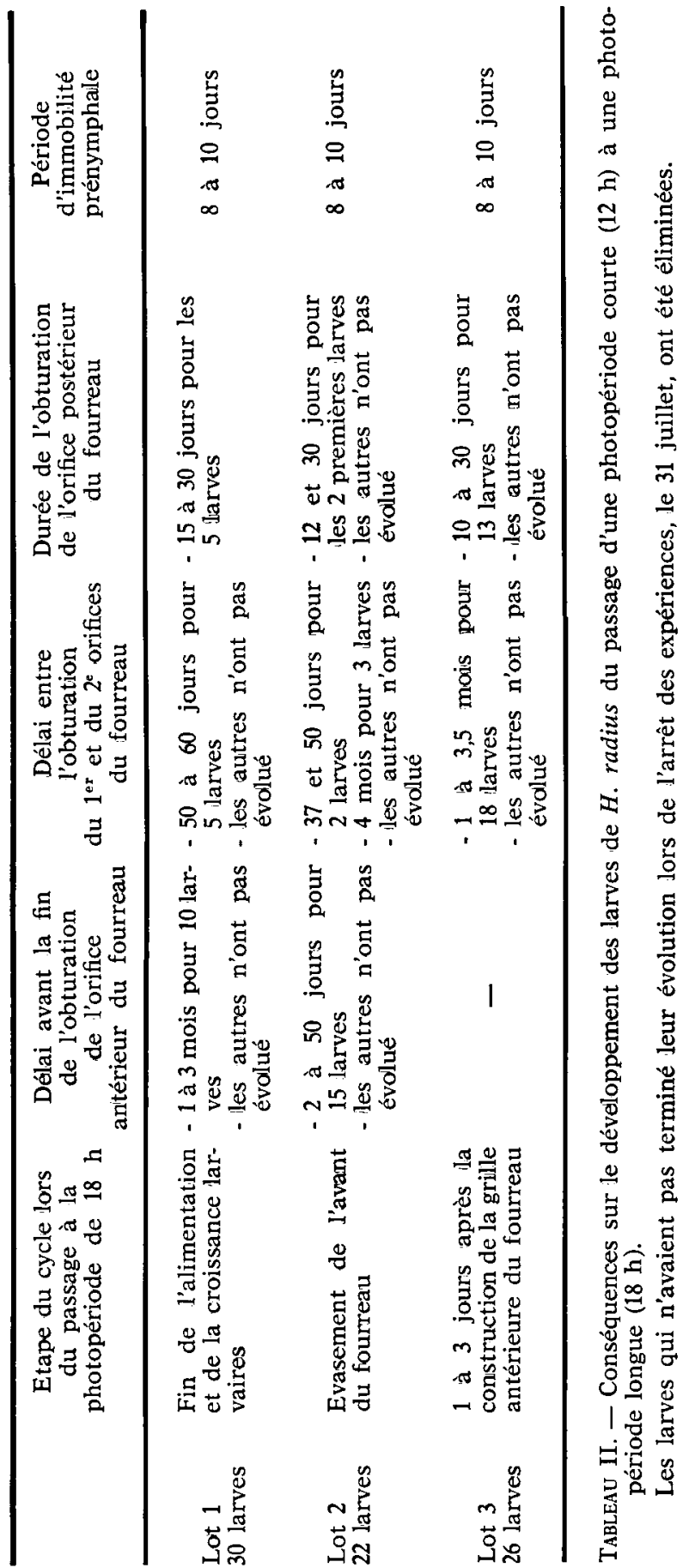


- a un effet freinateur presque immédiat,

- n'a plus d'effet sur la chronologie du développement, lorsque la grille postérieure du fourreau est construite.

\section{6. - CONCLUSIONS}

Cette étude a permis de mettre en évidence les points suivants :

1) La chronologie des différentes étapes du comportement à la fin de la vie larvaire est, chez Anabolia nervosa, brève et déterminée dès l'amorce de la construction de la grille antérieure du fourreau. Par contre, chez Halesus radiatus, cette chronologie est toujours nettement plus longue et demeure très variable jusqu'à la réalisation complète de la logette nymphale.

2) A chaque étape du comportement correspond toujours un état d'évolution organique défini et identique chez les deux espèces.

3) Pendant la diapause, le développement de $A$. nervosa est interrompu, celui de $H$. radiatus est soit stoppé, soit seulement ralenti.

4) L'action freinatrice de la photopériode s'exerce à un moment déterminé du cycle de $A$. nervosa : à la fin de la croissance de la larve et de son fourreau. Chez $H$. radiatus, cette action se manifeste à des mament différents du cycle: depuis la fin de la croissance de la larve et de son fourreau et jusqu'à la réalisation complète de la logette nymphalle.

Donc chez $A$. nervosa, la diapause se situe toujours à la fin du développement larvaire et stoppe complètement l'évolution des animaux. Chez $H$. radiatus, la diapause peut intervenir à des moments différents du cycle : dès la fin du développement larvaire mais également après le début de la métamorphose. Cela dépend de l'état d'évolution auquel les larves sont parvenues au moment où la photopériode devient inhibitrice, mais cela dépend aussi des individus car si l'évolution de certains est stoppée pendant la diapause, chez d'autres, elle se poursuit très lentement.

\section{TRAVAUX CITES}

Allegret (P.) et Denis (C.). 1972. - Dispositif pour l'élevage d'insectes aquatiques à température constante. Ann. Hydrobiol., 3 (I) : 65-7.

DENIs (C.). 1972. - Etude au laboratoire du cycle biologique de Anabolia nervosa (Trichoptera, Limnephilidae). Bull. Soc. Scient. Bretagne, 47 : 43-8.

DENIs (C.). 1973. - Influence de la photopériode sur le cycle biologique de Halesus radiatus (Limnephilidae). Bull. Soc. Scient. Bretagne, 48 : 193-6. 